\title{
Crashworthiness through smart use of high failure strain steel
}

\author{
M. M. H. H. Janssen, J. H. A. Schipperen \& A. W. Vredeveldt \\ Structural Dynamics, TNO, The Netherlands
}

\begin{abstract}
The crashworthiness of a structure can have a large effect on the area based risk in case of collisions. Typical examples are chemical tankers sailing the European inland waterways and offshore structures at our shores. Therefore, the assessment of crashworthiness is nowadays often included in the design process of such structures. But what if the design needs to be improved to meet the crashworthiness requirements? This paper describes a method to improve the crashworthiness of a ship structure by a smart use of high failure strain steel. It shows how the use of such material can be effective. High failure strain steels are normally more expensive than normal shipbuilding steels, so an optimization of the use of these materials is required. Therefore, a methodology is developed to determine the critical structural part(s) that would benefit most from the application of such steels. An example case of an inland waterway chemical tanker is given to illustrate the procedure. In this example, the crashworthiness of the design is determined through explicit FEM calculations. An elasto-plastic material model is used and material failure is included using a through thickness failure criterion with element deletion. Since this criterion was developed for mild steel it had to be adapted and validated for high failure strain steel using lab tests.
\end{abstract}

Keywords: crashworthiness, high failure strain, maritime structures.

\section{Introduction}

In this research the crashworthiness of a structure is defined as its ability to resist an impact without harming its occupants and environment. The unit in which the crashworthiness is often measured is energy [J]. The crashworthiness does not only depend on the structure but also on the characteristics of the impacting 
object and the location of impact. It is therefore good to realise than one does not determine the crashworthiness of a structure but the crashworthiness of the structure in a certain scenario. A crashworthiness assessment is often part of a risk assessment. The area based risk can in such cases be decreased by improving the crashworthiness of the structure for the impact scenario that causes the risk.

To understand how one can improve the crashworthiness it is important to understand the energy absorption mechanism of structures. During a collision most of the kinetic energy of the impacting object is dissipated through structural deformations. The crashworthiness of a structure increases when more material deformation can occur before the structure fails beyond a critical limit. This can be accomplished by adding more material to the structure or by the use of a material that can deform more before rupturing. Adding more material to the structure is often an undesirable solution since this leads to a heavier design and extra material costs. There exist steels that can deform more before failing than regular mild steels, referred to as high failure strain steels (HFSS) in this paper. Austenitic steel is an example of high failure strain steel, but even mild steel of which the failure strain is guaranteed to be higher than one would expect normally can be considered a high failure strain steel. This paper describes a method to improve the crashworthiness of a structure by smart use of high failure strain steel. An example case of an inland waterway chemical tanker is given to illustrate the procedure. In this example case the crashworthiness is determined by explicit finite element (FE) simulations.

First, it is explained how the use of high failure strain steel improves the structural crashworthiness. High failure strain steels are normally more expensive than normal shipbuilding steels, so an optimization of the use of these materials is required. To include the failure properties of the high failure strain steel in a FE calculation correctly, a failure model is needed. For this purpose a mild steel failure model is adapted and validated against results of drop tests on high failure strain steel strips. Finally, results are discussed and conclusions are drawn based on an example case in which the crashworthiness of a chemical tanker as shown in Figure 1 is improved.

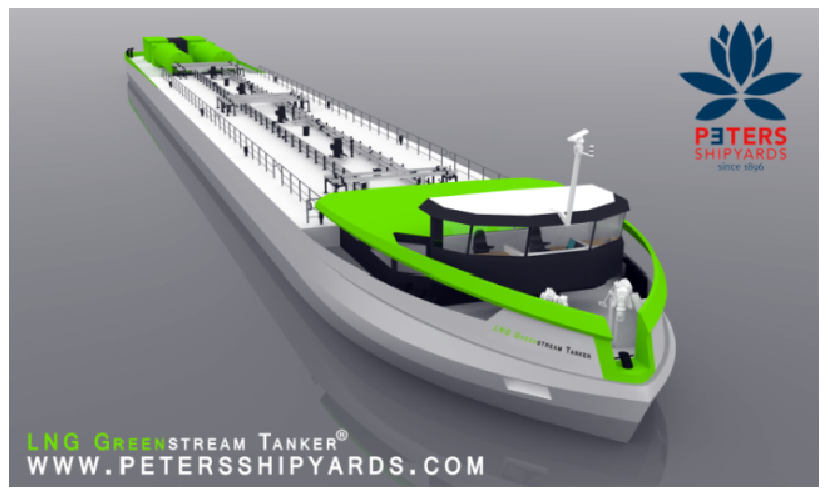

Figure 1: $\quad$ LNG greenstream tanker. 
The crashworthiness of this tanker has been increased because its hazardous cargo tanks exceed the maximum regulatory volume of $380 \mathrm{~m}^{3}$. The ADN rules [1] allow a vessel to sail with larger cargo tanks if it is proven that the associated risk does not increase compared to tankers complying with the $380 \mathrm{~m}^{3}$ requirement. The increase of the effect of outflowing cargo due to larger tanks can be compensated by reducing the probability of cargo outflow. This can be achieved by providing protection of tanks against collision through crashworthiness.

\section{How HFSS improve crashworthiness}

During an impact, the impacting object strikes the structure with a certain amount of kinetic energy. The amount depends on the impacting mass and velocity. This kinetic energy is mostly dissipated through material deformation and friction. Ductile materials, i.e. materials with a large failure strain, can deform more before tearing than materials with a smaller failure strain and can therefore absorb more energy. Figure 2 shows the stress versus strain curves for two different types of steel; mild steel and austenitic steel. The surface under the curve represents the amount of energy that is dissipated during deformation before material failure. The austenitic steel will absorb slightly more than two times the amount of energy that mild steel will absorb in a uniaxial tensile test, due to its larger failure strain of $41 \%$ compared to the $21 \%$ of the mild steel. The energy absorption due to elastic deformation is negligible compared to the amount of energy dissipated during plastic deformation. The larger energy absorbing capacity of high failure strain steels can be exploited to increase the crashworthiness of structures.

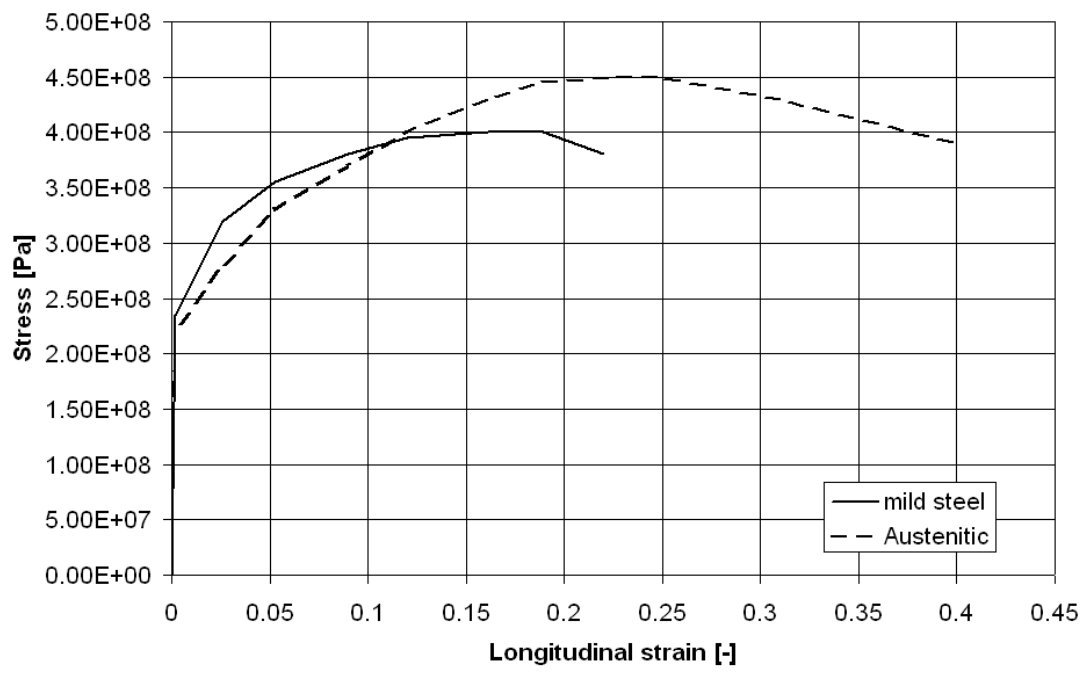

Figure 2: $\quad$ Stress-strain curve. 


\section{Smart use of HFSS}

High failure strain steels are normally more expensive than normal shipbuilding steels, so an optimization of the use of these materials is required for an efficient solution. To apply these steels in the most effective way one has to analyse the structure and its specific failure modes. The methodology to optimise the use of HFSS is described here:

Step 1: Selection of failure scenario(s) to improve

Which failure scenario is most likely to occur? And which scenario causes the largest risk for the environment? Most of the times these questions are already answered in a risk assessment and most likely a negative outcome of the risk assessment is the reason to improve the crashworthiness of the structure. The choice of the calamities and the relevant effects is application dependent and relies heavily on the judgment of the designer and the certifying authorities.

Step2: Analyse deformation during crash scenario

If the scenario for which the crashworthiness has to improve is known, one has to analyse which parts of the structure deform and fail before the event causes unacceptable damage. This can be done by reasoning, but a FE simulation of the crash scenario is often useful to confirm the occurring deformation.

Step3: Select structural parts to be produced from HFSS

Parts that deform heavily and lead to unacceptable damage when failed should be produced from HFSS to increase the crashworthiness. Parts that deform heavily but do not directly lead to unacceptable damage can be produced from HFSS, but the influence on the total crashworthiness is less. Costs should be weighted against the effect. Producing parts that exhibit only small or no deformations from HFSS does not increase the crashworthiness. If this is understood, the most optimal application of HFSS can be determined.

Step 4: Choice of HFSS

There are different sorts of HFSS. All steels with a higher failure strain than regular mild steel $(21 \%)$ are qualified as HFSS. The choice of HFSS depends on the desired crashworthiness improvement, the costs and the possibility to include the HFSS in the production process.

As an example a situation is taken in which an offshore platform is not sufficiently resistant to a collision with a supply vessel. The unfortunate event that can result from this situation is progressive collapse of the entire platform. It is known that this can occur when one of the four legs fails or one of the braces between two legs fails. Since the legs are crashworthy enough to resist an impact with a supply vessel but the braces are not, the braces are the most critical parts. If the braces are produced from high failure strain steel the braces will absorb more energy before failing and the crashworthiness of the structure is improved. Another example is discussed more elaborately in chapter 5.

The deformation of a structure as a result of an impact can sometimes be estimated analytically but for complex structures explicit dynamic FE analyses are nowadays common practice. The crashworthiness of the example in this research is determined using the explicit FE code LS-Dyna. Material failure is included by the use of a material failure criteria with element deletion. The next 
section discusses the failure criterion that is used in this research and the adaptions that had to be made to make the criterion suitable for the use with high failure strain steel.

\section{Prediction of HFSS failure in FE analyses}

To describe the correct failure behavior of high failure strain steel a mild steel failure model developed by GL and described in the ADN rules [1] was adapted. The mild steel failure model is a combination of an elasto-plastic material model with a through thickness failure criteria with element deletion. The failure criterion is described by:

$$
\varepsilon_{f}\left(l_{e}\right)=\varepsilon_{g}+\varepsilon_{e} \frac{t}{l_{e}}
$$

where $\varepsilon_{\mathrm{f}}$ is the longitudinal failure strain, $\varepsilon_{\mathrm{g}}$ is the structural uniform strain, $\varepsilon_{\mathrm{e}}$ is the necking strain, $t$ is the element thickness and $l_{e}$ is the characteristic element length. The values for the structural uniform strain and the necking strain depend on the used material and the geometry of the structure. Classification society Germanischer Lloyd has performed measurements on real ship structures which were damaged in collisions. Thickness reductions on different distances from the rupture were measured and the values to be used for the structural uniform strain $\left(\varepsilon_{\mathrm{g}}=0.056\right)$ and the necking strain $\left(\varepsilon_{\mathrm{e}}=0.54\right)$ were derived from these measurements. Since the values are based on measurements performed on real deformed ship structures the effects of welds, slight deformations and the materials stress state during deformation are included. The longitudinal failure strain element deletion criterion in the material model used in LS-Dyna does not distinguish between strain in compression and tension. This means that, when using this option in LS-Dyna, elements which are compressed and become thicker fail, while in practice this is not the case. Therefore, a through thickness strain is used instead as a failure (element deletion) criterion in which elements fail after a certain thinning of the material. Under the condition that the plate is constrained in transverse direction and that the total volume remains constant the critical thickness strain becomes:

$$
\varepsilon_{t}=\frac{\varepsilon_{f}}{1+\varepsilon_{f}}
$$

In case of $l_{e} / t$ ratios smaller than 5 , it was seen in previous analyses that the energy absorption of structures modeled with these elements increased unrealistically. Hence, an improved formula for the through thickness failure strain was derived for those elements exceeding the $l_{e} / t<5$ ratio, leading to more consistent energy absorption results [2]:

$$
\varepsilon_{t}=0.27\left(\frac{l_{e}}{t}\right)^{-0.4163}
$$

The through thickness failure strain is plotted against the element length over thickness ratio in Figure 3. 


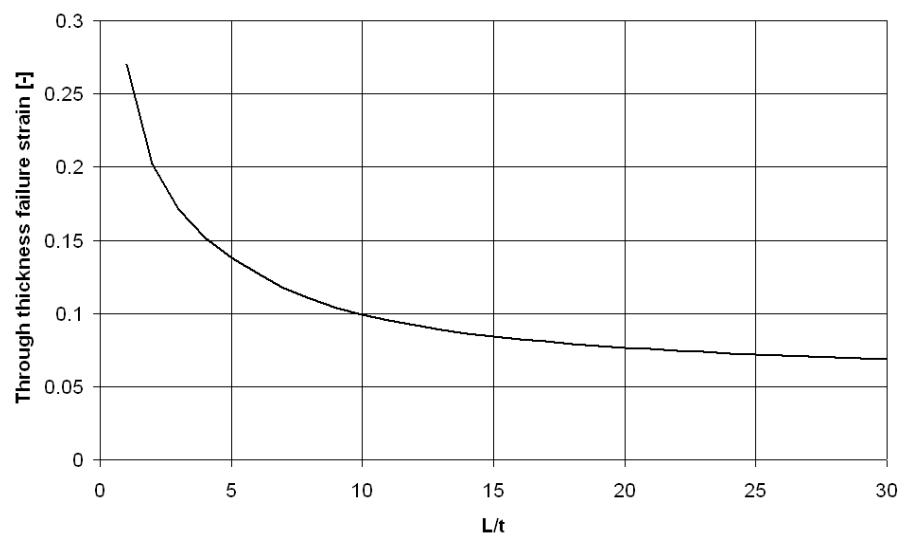

Figure 3: Through thickness failure strain as function of $l_{e} / t$.

This failure criterion was validated by full scale collision tests performed by TNO as part of the European research project Crashcoaster [3]. The contact force between the two colliding vessels was measured and compared with simulation results. Figure 4 shows that the predicted force agrees well with the measured force.

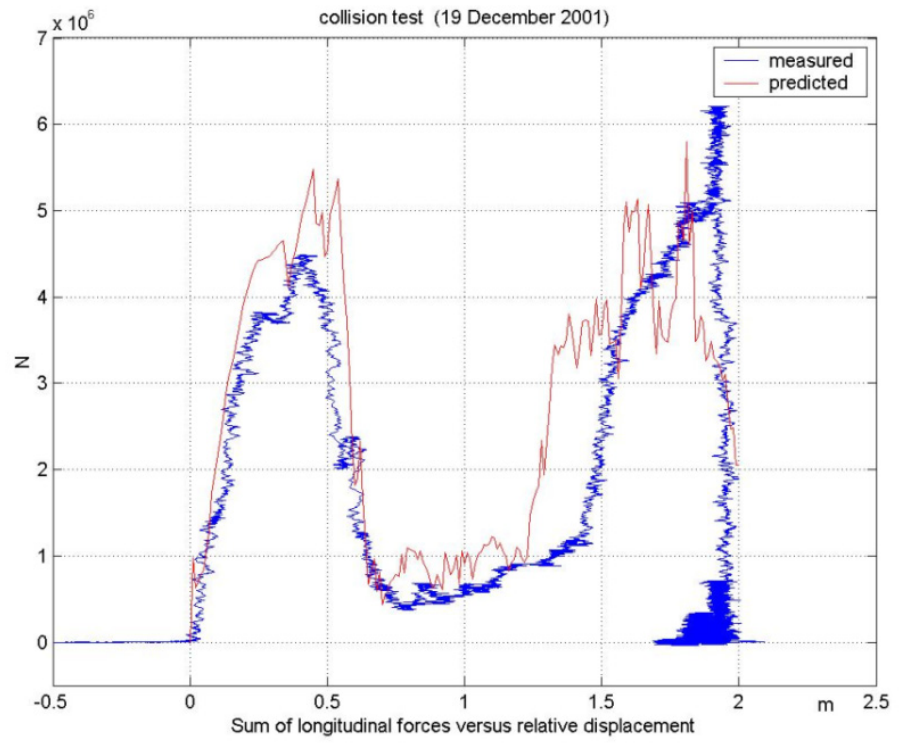

Figure 4: Contact force measured during full scale experiment compared with contact force predicted by explicit FE simulation. 
Since the parameters of the failure criterion are based on measurements on real damaged ship structures made from regular mild steel, the parameters are not suited to predict the failure of a ship structure made from high failure strain steel.

The criterion (eqn (1)) has to be adapted.

Drop tower tests on mild and austenitic steel showed that both the structural uniform strain $\left(\varepsilon_{\mathrm{g}}\right)$ and the necking strain $\left(\varepsilon_{\mathrm{e}}\right)$ increase with increasing longitudinal failure strain [4]. Because the ratio between the longitudinal failure strain of mild steel and high failure strain steel can be easily determent by a tensile test it was decided to use this ratio to scale the longitudinal failure strain used in FE simulations. It is assumed that the structural uniform and necking strain show similar dependence on the longitudinal failure strain. The adapted failure criterion is given by eqn (4):

$$
\varepsilon_{f}\left(l_{e}\right)=\frac{\varepsilon_{h f s s}}{\varepsilon_{m s}}\left(\varepsilon_{g}+\varepsilon_{e} \frac{t}{l_{e}}\right)
$$

where $\varepsilon_{\mathrm{hfss}}$ is the elongation at failure of the high failure strain steel and $\varepsilon_{\mathrm{ms}}$ is the elongation at failure of mild steel determined from uniaxial tensile tests.

Note that the longitudinal failure strain in an uniaxial tensile test is probably larger than the longitudinal failure strain in a structure. This is not only due to the presence of welds and slight deformations but also due to the stress state of the material. Under bi-axial loading, which is the typical stress state in a side shell of a vessel during a collision, the longitudinal failure strain is significantly smaller than under uniaxial loading [5]. However, it is assumed that the ratio between the longitudinal failure strain of mild steel and HFFS is not affected by the stress state.

To test if this adapted criterion yields reasonable results in FE analyses drop tower tests done on long uniaxially loaded specimens [4] are simulated. Figure 5 shows the predicted and measured thickness reduction of the mild steel (MS) and austenitic (Aust.) plates after testing. The simulations using the adapted failure criterion give under-predictions for both the mild steel plates and the austenitic plates with regard to the amount of deformation and energy absorption. This was already expected since the plates in the drop tower tests are loaded uniaxial while the parameters of the failure criterion are based on a more bi-axial loading state. Besides that, the tested plates are not affected by welds and irregularities. The ratio between the energy absorption of the two different materials is correctly predicted. In both the experiments and simulations, it was found that the austenitic plates absorb about 2.4 times the amount of energy than the mild steel plates absorb before failure. This corresponds well with the expectations based on the surface under the stress strain curves shown in Figure 2.

The performance of the for HFSS adapted failure criterion in the drop test simulation is comparable to the, for ship to ship collisions validated, original mild steel failure criterion. It is therefore valid to state that the, for HFSS adapted, criterion is able to correctly predict the amount of energy absorption in a ship to ship collision. 


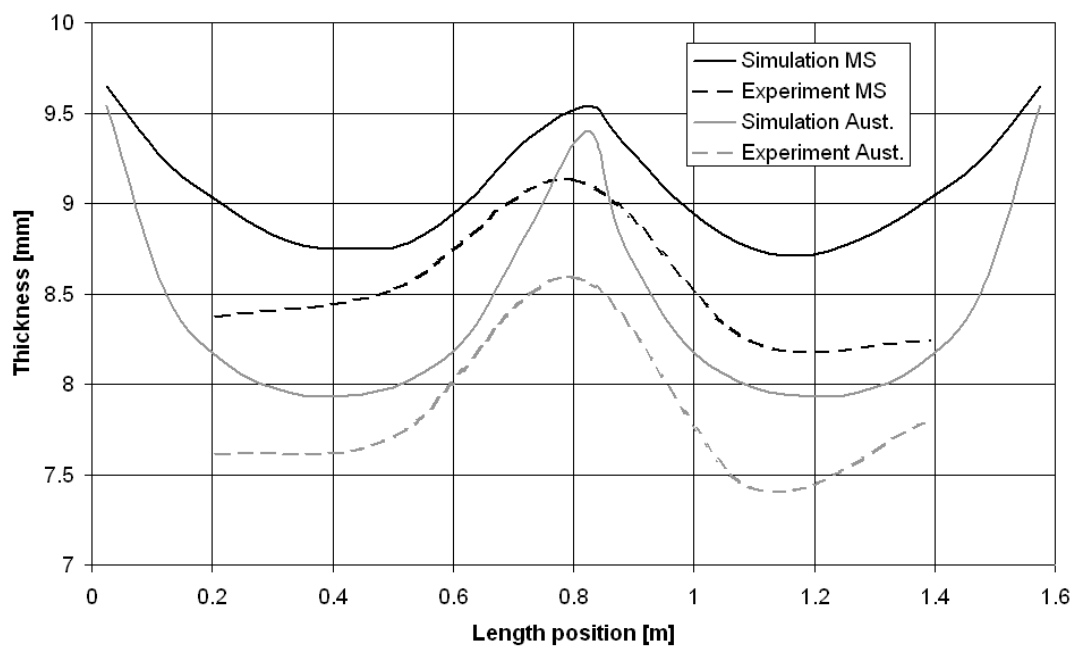

Figure 5: Measured and predicted thickness over the length of the specimen after drop tower test. Original thickness $10 \mathrm{~mm}$. Thickness at position 0 and $1.6 \mathrm{~m}$ not measured in experiment.

\section{Example}

In the example case the crashworthiness of the chemical tanker shown in Figure 1 is increased because its hazardous cargo tanks exceed the maximum regulatory volume of $380 \mathrm{~m}^{3}$. The ADN rules allow a vessel to sail with larger cargo tanks if it is proven that the associated risk does not increase compared to tankers complying with the $380 \mathrm{~m}^{3}$ requirement. The effect increase at tank rupture due to larger tanks can be compensated by reducing the probability of cargo outflow. This can be achieved by providing protection of tanks against collision through crashworthiness. The probability of cargo outflow can be calculated by following the rules described in [1]. The crashworthiness of the hull design is evaluated under typical collision scenarios, comprising of:

- $\quad$ Two impacting bow designs (push barge bow and V-bow)

- Four typical horizontal impact locations

- Five typical vertical impact locations

The collision with a push barge bow is at an angle of $55^{\circ}$, collision with the $\mathrm{V}$-shape bow at $90^{\circ}$. Because not all scenarios can be discussed in this paper only the scenario with the largest probability of occurrence is given. Note that the material design adaptations not only contribute to the crashworthiness of this scenario but also to the crashworthiness of other scenarios. The steps that result in a smart use of HFFS are described below.

Step 1: The scenario with the largest probability of occurrence is a collision between the chemical tanker and a push barge. Based on the draught of the chemical tanker and the draught of the typical push barge it is most likely that a 


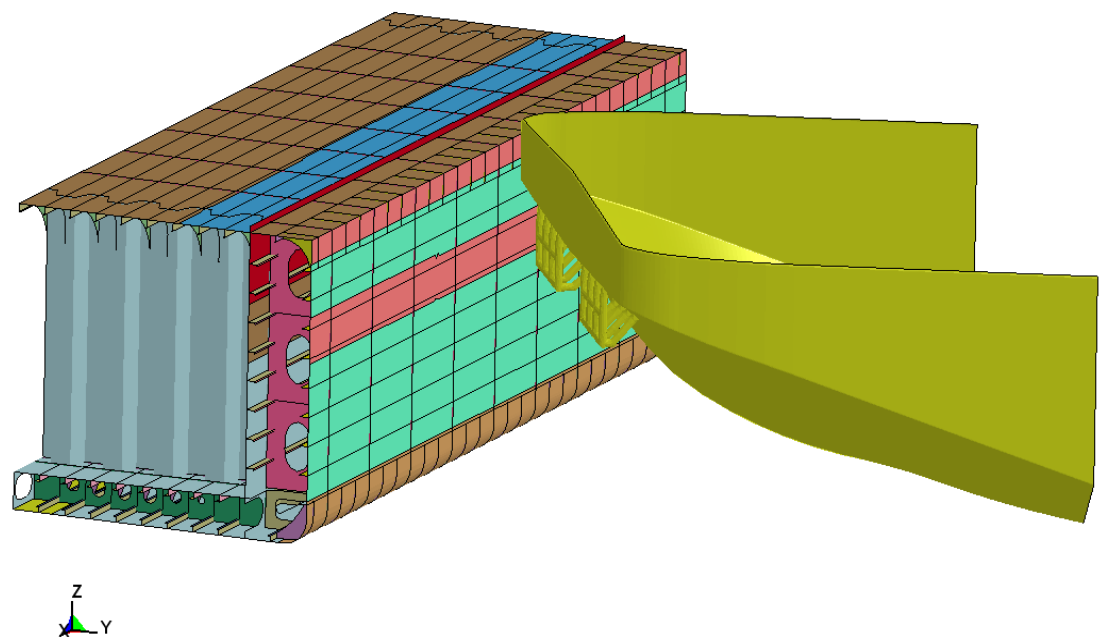

Figure 6: $\quad$ FE model of the chemical tanker and the striking push barge bow.

push barge strikes the chemical tanker at a location as shown in Figure 6. A cargo spill occurs when the inner shell of the vessel tears.

Step2: In this example, the deformation during the crash is analyzed using an explicit FE calculation. The FE model, as shown in Figure 6, represents one tank compartment of the chemical tanker. The length of the compartment is $17 \mathrm{~m}$. To reduce the required CPU time only half of the width of the tanker is modeled, i.e. from the side shell to the centerline. The movement of the compartment ends is restricted and the velocity of the striking push barge bow is prescribed. The model consists of 249.746 elements. The element type that is used is a four node Belytschko-Tsay shell element with 5 integration points through the thickness. The focus of this model is to predict the amount of energy absorption before tank rupture due to deformation of the structure. The global dynamic movement of the vessel due to the collision is not included.

The FE simulation shows that the inner shell of the vessel tears at the moment the striking push barge bow has penetrated the chemical tanker by $1.72 \mathrm{~m}$. The amount of energy absorbed by the structure is then 8.7 MJ. Figure 7 shows the extent of the plastic deformation of the ship structure at a moment just prior to tank failure. All parts which are coloured are plastically deformed. Figure 8 shows the amount of plastic deformation and the failure mechanism of the inner shell. It can be seen that the shell fails next to a deck girder. Before failing, the shell is loaded in biaxial tension. The shell fails next to the deck girder because the deformation at that location is locally constrained by the presence of the deck girder.

Step3: The crashworthiness of this scenario can be improved by replacing a strip of conventional mild steel at the top of the inner shell with a strip of high failure strain steel. Since analyses showed that in most of the collision scenarios analysed for the full risk assessment the tank ruptured at the top part of the inner 


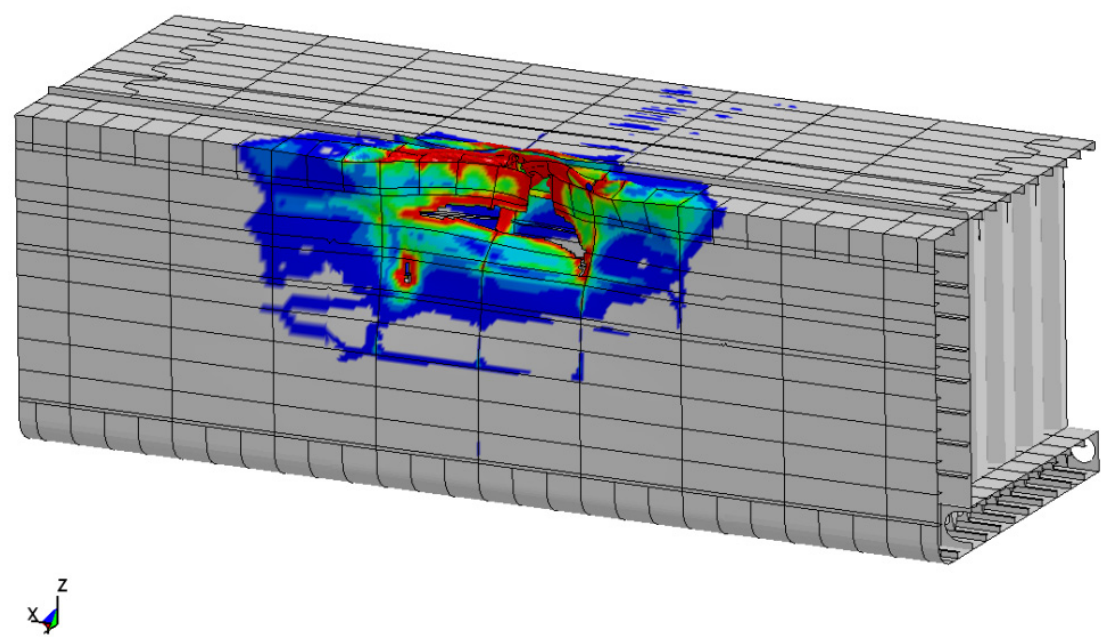

Figure 7: Plastic deformation of the chemical tanker just prior to tank failure.

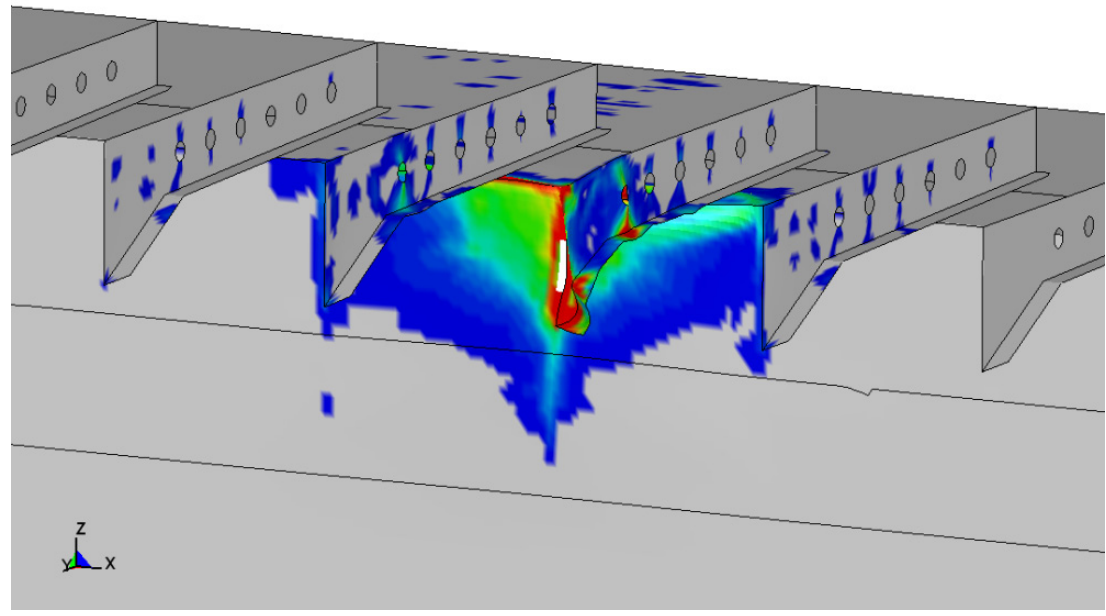

Figure 8: Plastic deformation of the inner shell and first material failure. View from tank side.

shell or at the deck it was decided to also replace a strip in the deck with a strip of high failure strain steel.

Step 4: The type of high failure strain steel which is used to improve the crashworthiness in this example is S235JR with a guaranteed minimum failure strain of $37 \%$. This material is chosen because the application of this material in the production process of the vessel does not require any adaptations. The 
material can be welded and cut like conventional mild steel. Besides that, the costs are only slightly higher than the costs of conventional mild steel.

To be able to calculate the adapted failure strain one also has to know the failure strain of mild steel. According to Lloyd's [6] mild steel has a minimum guaranteed failure strain of $21 \%$. However, tensile tests show that the average value is about $31 \%$. One can argue about whether to use a value of $37 / 21$ or $37 / 31$ to scale the longitudinal failure strain. For this research it was decided to be conservative and use the factor $37 / 31$ leading to $18 \%$ more energy absorption in a uniaxial tensile test. The assumed yield stress and hardening curve of the S235JR steel are the same as for regular mild steel.

The FE calculations of the improved design show that the inner shell ruptures after a penetration depth of $1.82 \mathrm{~m}$. The failure mechanism of the inner shell has not changed. Rupture occurs next to the deck girder. The structure has absorbed 9.8 MJ. This is an increase of $13 \%$.

For the chemical tanker, the use of high failure strain steel was one of the structural adaptations that made the structure more crashworthy than comparable chemical tankers that comply with the $380 \mathrm{~m}^{3}$ rule. The use of high failure strain steel to improve the crashworthiness was a relatively cheap and easy measure which did not increase the mass of the vessel. It is determined, following the procedure described in the ADN rules [1], that due to the $13 \%$ increase of the crashworthiness the probability of tank failure in a collision is reduced by $20 \%$. The chemical tanker complies with the rules due to the effective use of high failure strain steel.

The increase in energy absorption is less than the ratio between the failure strains (37/31) because most energy is dissipated by deformation in the outer shell and webs before the inner shell even starts to deform. Deformation of the inner shell starts at a penetration depth of about $1.28 \mathrm{~m}$. Nevertheless, producing the outer shell and webs from high failure strain steel would probably not have increased the crashworthiness. Since, whether or not the material of the outer hull fails it continues to deform and absorb energy till the inner shell ruptures.

\section{Conclusions}

It is shown how the use of high failure strain steels can improve the crashworthiness of a structure and a method for smart usage of this material is described. The effectiveness of the use of high failure strain steel depends on the contribution of the in high failure strain steel produced part to the total crashworthiness and on the stress state of that part. In complex structures, such as ship structures, it is hard to estimate in advance the effect of HFSS on the crashworthiness. Therefore, FE analyses are necessary. The failure criterion that predicts material failure in the FE analyses in this research is adapted to be used with high failure strain steel. It is shown that the longitudinal failure strain can be scaled with the ratio between the elongation of the high failure strain steel and the elongation of the regular mild steel determined in a uniaxial tension or drop tower test. In the example case, the crashworthiness of the chemical tanker 
increases by $13 \%$. Due to a $13 \%$ increase of the crashworthiness the probability of tank failure in a collision is reduced by $20 \%$.

\section{References}

[1] Vredeveldt, A.W., Zentralkommission für die Rheinschifffahrt, Anlage zum Europäischen Übereinkommen vom 26. Mai 2000 über die internationale Beförderung von gefährlichen Gütern auf Binnenwasserstraßen (ADN), Chapter 9.3.4.3 Berechnungsverfahren and Chapter 9.3.4.4 Ermittlung des Kollisionsenergie-Absorptionsvermögens, January 2011.

[2] Luyten, J.M., Afleiden faalrekken dikke platen voor verschillende elementgroottes, internal memorandum TNO, 2011.

[3] Vredeveldt A.W., Hacken ten R., Crashcoaster: Full scale collision experiment, conventional double hull structure, general cargo casco, TNO report 2002-CMC-R009, 2003.

[4] Pel, S, Behaviour of mild steel and austenitic steel under impact loading, TNO report 2007-D-R0084/A, 2007.

[5] Wierzbicki, T., Bao, Y., Lee, Y.., Calibration and evaluation of seven fracture models, International Journal of Mechanical Sciences 47, pp. 719743, 2005.

[6] Lloyd's Register, Rules for the manufacture, testing and certification of materials (Chapter 3), Rolled steel plates, Strip, Sections and Bars, 2009. 